\title{
Structural Modal Testing Using a Human Actuator
}

\author{
Ziping Han ${ }^{1}$, JMW Brownjohn ${ }^{2}$, Jun Chen ${ }^{1 *}$ \\ 1. Department of Structural Engineering, Tongji University, Shanghai, China
}

2. College of Engineering, Mathematics and Physical Sciences, University of Exeter, United Kingdom

\begin{abstract}
The dynamic testing of as-built structures is essential for understanding structural dynamic properties, updating finite-element models, maximizing the effectiveness of structural performance monitoring, and planning vibration mitigation measures. Traditional dynamic test technologies using logistically challenging systems of mechanical actuators, sensors, and signal generation/acquisition instruments may not be feasible for the modal testing of low- to mediumrise buildings, which comprise the majority of commercial and residential buildings in cities.

The human body, which is an intelligent dynamic system with advanced sensing and control abilities, has demonstrated excellent potential as an excitation source for structure vibration owing to individuals and crowds walking, jumping, bouncing, or swaying. The force generated by human activity can be maintained over a narrow frequency bandwidth and can reach significant amplitudes, readily generating the resonance response of structures with low natural frequencies.
\end{abstract}

This study investigates the use of humans as actuators for structure modal testing. The application protocol is first proposed, and intelligent wearable sensors and force plates are used to calibrate humans as actuators. By modelling human as combination of rigid bodies, with an appropriate choice of mass participant ratio, the human exciting force can be well reconstructed using the acceleration measured by wearable sensors at featured body points. The application of the proposed protocol on a building with a typical frame construction and on a large span floor verified its suitability for estimating the modal frequency, damping ratio and modal mass

\footnotetext{
${ }^{*}$ Correspondence author
} 
with reasonable accuracy, thereby demonstrating the practicability of modal testing using human actuators.

Keywords: Human excitation, dynamic modal testing, wearable sensors, lateral acceleration, ground reaction force.

\section{Introduction}

To understand and interpret with confidence the actual performance of a structure, it is important to know its dynamic properties, such as natural frequencies, modal damping ratios, as well as modal mass. In many cases, it is also important to verify and update the numerical model used in the design stage (Friswell \& Mottershead, 2013; Doebling et al., 1996) in order to develop better design strategies for future similar structures, and for informing and assessing structural retrofit. Moreover, to address vibration serviceability problems of as-built structures, avoiding structural damage or enhancing the benefit of long-term structural health monitoring, estimations of structural modal properties (modal frequencies, shapes, masses, and damping ratios) are necessary (Doebling et al., 1996), and they provide significant motivation to carry out vibration testing. Various techniques of experimental modal analysis can be employed to identify the structural dynamic properties, which specifically include the modal mass (or mode shape scaling), whose value is needed for response prediction, or (inverse) load estimation. Classified by the excitation source, several common technologies are employed for dynamic testing; these include shaker testing, hammer testing, ambient vibration testing, and free vibration testing (Ewins D J, 1984).

The ambient vibration test (AVT) is commonly used for large civil structures such as longspan bridges (Chen \& Xu, 2003), high-rise buildings (Ventura et al., 2002), and large grandstands (Reynolds et al., 2005), which are all very difficult to be excited by dynamic actuators, whether for technical or cost reasons. The AVT treats wind, traffic, or other 
environmental excitations as inputs, and measures structural responses only. Structural modal parameters are identified by assuming the excitation as a white-noise process. Considering that global vibration modes are usually sufficiently excited under the normal surrounding conditions (Saudi et al., 2009), AVT is an appropriate procedure for the modal testing of lower modes of large structures. However, due to the random properties of the ambient loading, there is greater uncertainty inherent in modal parameter estimation using AVT data is small compared to forced vibration testing, especially for damping ratios (Au, 2018).

Compared with AVT, the use of shaker (actuator) or impact hammer testing may provide both the excitation and response information over a wider exciting bandwidth, and the ability to control and measure the forcing function enables the most reliable modal analysis. Hammer testing has a lower logistical demand, but the disadvantages include the reduced ability to control the force bandwidth and the very high signal crest factors, which may overload data acquisition systems; a shaker is thus preferred. However, most actuators are generally logistically challenging to deploy due to size, weight, power requirement, and in extreme cases, requirement for local structural modification. Therefore, they are more likely to be required when high-quality information, including modal mass/mode shape scaling, is required. Such a requirement usually exists for more important structures, where response simulations rely on modal parameter estimation. The force that is generated by a shaker, whether it is a linear hydraulic, electromagnetic, or rotating mass, is proportional to its mass, range of mass movement (stroke), and the square of vibration frequency. Therefore, a large mass and/or longstroke multiple shakers are necessary to test large civil structures (with low frequency). Such shakers usually need to be custom-built at considerable expense (Severn, R. T., 1997, Stoessel J C et al., 1986) and hence the shaker testing of very low-frequency structures such as long span bridges or tall building is extremely rare. In fact, the few historic applications to tall buildings and long-span bridges rarely recorded the force signals with their phase angles, limiting the 
reliability of modal parameter estimates (Okauchi et al., 1986; Ohlsson, 1987). The major benefit of generating large and controlled artificial forces has been to study the nonlinearity and to obtain a reliable estimation of the damping and frequency from single-mode free decay, although modern operational modal analysis methods have now largely surpassed the capabilities from free vibration, such as stochastic subspace iteration (Peeters, et al., 2007) and Bayesian operation modal analysis (Au, 2011). For the large number of low-rise, medium-size buildings with modal masses of the order of $10^{4}$ metric ton, shaker testing may not be feasible in terms of test costs, so there is a need for a more efficient forced vibration test method that is applicable for various types of structures and different test conditions.

Exciting vibrations using human force is particularly common in the study of the vibration serviceability of floors, footbridges, and stadia. It is believed that the first recorded test using crowd swaying and stepping was in the Wembley Stadium (Wembley, 1923), and a common but somewhat discredited method for checking the floor vibration serviceability is the heel drop test (Lenzen, 1966). As an example, when testing a floor, the tester stands on the floor center, rises on his/her toes, and then drops heels down to strike the floor. The floor deformation time history under this heel-drop impulse is measured and compared with numerical results to identify the floor modal characteristics (Blakeborough \& Williams, 2003). This method formed a part of many design procedures owing to its efficiency and operability (Allen, 1974; Canadian Standards Association, 1994; Murray, 2003; Willford et al., 2006; Smith et al., 2009), but is not employed in more advanced criteria used in Europe (Feldmann et al., 2010). Modern large civil structure designs favor spans that are more lightweight, taller, and longer owing to the widespread application of new construction material and technologies, and these can be excited to significant and uncomfortable vibration levels by human activities, as happened at the London Millennium Bridge (Dallard et al., 2001) and Techno-Mart Building (Lee et al., 2013). As large crowds walked on it during the bridge's opening day, it vibrated excessively, and had 
to close for three years to facilitate adequate investigations and retrofitting. The Techno-Mart Building is a 39-storey high-rise in Seoul, South Korea, which vibrated for more than 10 min owing to an exercise activity by 17 adults in a gym on the 12th story.

Although they are normally treated as engineering accidents, these examples indicate that the same type of human activity that caused the problem can also be used to excite it. For example, based on the authors' experience (Chen et al., 2015), jumping by eight persons can excite a $90 \mathrm{~m}$ (side length) by $30 \mathrm{~m}$ (span) concrete floor to $4 \mathrm{~cm} / \mathrm{s}^{2}$ acceleration response (peak of 10-s running root mean square).

The human body is an intelligent biological dynamic system with advanced sensing and control abilities, and a healthy adult is capable of generating low-frequency (normally $0.25 \mathrm{~Hz}-$ $5.0 \mathrm{~Hz}$ (running)) excitation that is sufficiently stable and long lasting to excite a structure into resonance. Thus, humans are potential actuators for most structures with low natural frequency, such as high-rise buildings (Lee et al., 2013, Glanville et al., 1996), long-span footbridges (Brownjohn \& Pavic, 2007, Sadhu et al., 2019), stadia (Reynolds et al., 2005), and large span floors (Pavic \& Reynolds, 1999). For example, experiments on a full-scale floor with different dynamic loads, including human walking, sandbag impact, and hammering, were investigated by Zhou et al. (2014), and their study showed little difference in natural frequency estimates among the methods. Metronome-prompted walking is now a common procedure during vibration serviceability checks for footbridges and floors (Pavic \& Reynolds, 1999; Brownjohn et al., 2016); tests implemented on four full-scale footbridges based on a human-induced load database gave an acceptable accuracy with an error margin of about $15 \%$ for modal mass estimation (Brownjohn \& Pavic, 2007). Impulsive loading due to a heel drop or one-time jump is commonly applied to generate free decay response, but without force data this provides no information about the structure's modal mass, which is needed for vibration serviceability assessment. 
Despite many attractive attributes of the human actuator for modal testing, the main problem is the difficulty of directly measuring the exciting force, and recording it synchronously with the structural responses. Instrumented force plates used in laboratory biomechanics research are unsuitable for field use and and there is a need for a single type of easy-to-use, and wireless sensor to simultaneously serve multiple purposes of human actuator indirect inertial force measurement and response measurement. Over the last decade, there have been rapid advances in the technology of microprocessors and sensors packaged into wearable/portable devices such as smartphones, smartwatches, fitness trackers, and wearable inertial measurement units (IMUs). Embedded three-axial accelerometers and gyroscopes can measure a device's acceleration and direction with sufficient precision and a sufficiently high sampling frequency for them to be suitable for use in motion monitoring and structural dynamic testing (Brownjohn \& Pavic, 2007; Chen et al., 2016, Wang et al., 2017, Brownjohn et al., 2018). This progress with wearable devices offers a novel and powerful means of overcoming the above two problems related to the application of human actuators.

This paper proposes a framework that uses human excitation for structure modal testing. Section 2 introduces the basic idea of the framework, with emphasis on the application procedure, reconstruction of the excitation force, and modal parameter identification. Section 3 discusses the selection of wearable sensors for the modal testing. Section 4 presents in detail the calibration of human actuators and the reconstruction of human-generated swaying and jumping forces. Section 5 applies the test method to two existing structures: an eight-story steel frame structure under swaying excitation and a long-span floor under jumping excitation. Finally, Section 6 discusses limitations of the human actuator testing method and concludes the main findings of this study. 


\section{Human actuator for structure model testing using wearable sensors}

\subsection{Proposed test protocol}

As mentioned above, by performing activities such as walking, jumping, running, bouncing, and swaying, the human body can easily generate substantial excitation in both vertical and lateral directions (Chen, 2016). The use of human actuators eliminates the logistical challenges of electromechanical shakers. Different from such shakers, the human exciting force is not constrained by the fixed-stroke oscillation of a mass. Instead of increasing (slowly) with the square of the oscillation frequency, it rises quickly from zero frequency and can be substantial, reaching several times the body weight. Based on the wearable/portable sensors employed, the test protocol for a human actuator for typical structures can be summarized as follows:

(1) Conduct a calibration test to understand the relationship between the tester's activity (e.g., swaying or jumping), its frequency, and the force generated.

(2) Obtain preliminary estimates of the natural frequencies of the test structure using a simple method such as AVT/heel drop using wearable sensors.

(3) Excite the structure at the frequency of the vibration mode of interest by the tester performing a suitable activity. Wearable sensors are used to measure simultaneously the tester's activity (the timing information of accelerations experienced by the tester's body) and the structure's responses.

(4) Reconstruct the excitation force using the measured tester's activity (accelerations) by employing relations gathered from the calibration test.

(5) Identify the structure's frequency, damping ratio, and modal mass using information of the input (excitation force in step 4) and output (responses measurement in step 3).

The following flowchart in Figure 1 summarizes the above test procedure using a human actuator. 


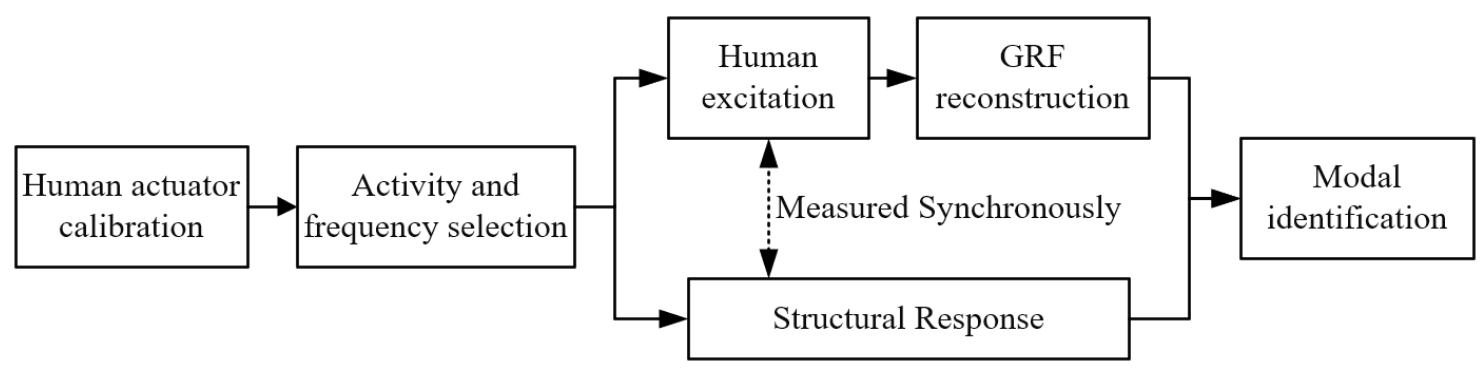

Figure 1. Procedure of structure model test using human excitation.

As a human actuator, one tester with one or more wearable portable sensors can quickly and easily perform comprehensive on-site testing of medium-sized buildings or the substructures (e.g. floors, staircases) of large, significant buildings.

\subsection{Reconstruction of excitation force}

A key step in the above test protocol is the reconstruction of the tester's excitation force for different activities using wearable sensor measurements. To this end, a rigid body model can be used to reconstruct the exciting force using accelerations of featured points of the human body (Racic et al., 2010, Zhang et al., 2013)

$$
\begin{aligned}
& \operatorname{GRF}_{v}(t)=\sum_{i=1}^{n} m_{i} R_{i v} a_{i v}(t)+G \\
& G R F_{l}(t)=\sum_{i=1}^{n} m_{i} R_{i l} a_{i l}(t)
\end{aligned}
$$

Where $G$ is the total weight of the human body, the ground reaction force (GRF) is the exciting force generated by human activities, $n$ is the number of segments of a human multi rigid body model (Aggarwal \& Cai, 1999). For each body segment, $m_{i}$ and $a_{i}(t)$ are the mass and acceleration of its feature point, respectively. $R_{i}$ is the mass participant ratio, which represents the proportion of the mass participating in human dynamic activity, and subscripts $v$ and $l$ indicate the vertical and lateral directions, respectively.

In the rigid body model, the number of body segments varies from one (Cheng et al., 2000) to several thousand (Pearsall et al., 1994), with a still ambiguous inertial mass and center-of- 
mass (COM) of each segment. From the perspective of its application, it is impractical to reconstruct the GRF using a model with multiple segments, so the following simplified model with only one segment is thus very often adopted:

$$
\begin{aligned}
& G R F_{v}(t)=M R_{v} a_{v}(t)+G \\
& G R F_{l}(t)=M R_{l} a_{l}(t)
\end{aligned}
$$

By assuming the human body as a single rigid body, which is commonly used in humaninduced load studies (Bobbert et al., 1991; Racic et al., 2010; Zhang et al., 2013;), only the total mass $M$, acceleration $a(t)$ at the COM and overall mass participant ratio $R$ is needed. As the movement of the body COM cannot be determined and measured directly, the acceleration of a feature point is applied to represent the actual COM of the body.

For structural dynamic modal testing, the human actuator must be calibrated in advance to identify a suitable feature point for acceleration measurement and the corresponding mass participant ratio. Then, one or several persons can excite the structure with the calibrated activity and pre-estimated frequency. Meanwhile, wearable sensors such as IMUs or smartphones are used to measure human body feature point's acceleration and structural response synchronously. Finally, using the reconstructed exciting force and structural response, the modal properties can be identified.

\subsection{Modal parameter identification}

After calibration, wearable sensors such as IMUs or smartphones can be used to measure the human body feature point's acceleration and structural response synchronously. Once the input (exciting force) and output (structural response) of the structure system are available, the modal frequency and damping ratio can be estimated using one of the many well-established methods. With respect to the modal mass, which is essential for response prediction as well as for designing passive vibration controllers such as tuned mass dampers or tuned liquid dampers 
(Housner et al., 1997), this study proposes an estimation method which assumes that the structure behaves as a single-degree-of-freedom (SDOF) system. Details of the parameter identification are provided in section 5, along with examples.

\section{Selection of wearable sensors}

Different types of wearable sensors, including various brands of smartphones (Chen et al., 2016) and wearable IMUs, were compared. Their performance characteristics, including the measurement accuracy and range, means of fasten and carry, modes of data transmission, and synchronization were seriously considered. The IMU Opal (Figure 2) produced by APDM Inc. was finally selected for this study, and it has also been used by many other researchers (Racic

et al., 2013; Bocian et al., 2016; Brownjohn et al., 2016). The Opal IMU contains a tri-axial accelerometer and gyroscope for the measurement of the acceleration and rotation angle, and the magnetometer helps with the alignment with the world coordinate system. It can transmit data to a computer in the $2.40-2.49 \mathrm{GHz}$ wireless range or save data in internal storage. Synchronization errors among different units are under $10 \mu \mathrm{s}$, which is sufficiently low for research on human-induced loading. Table 1 shows the main parameters of the Opal.

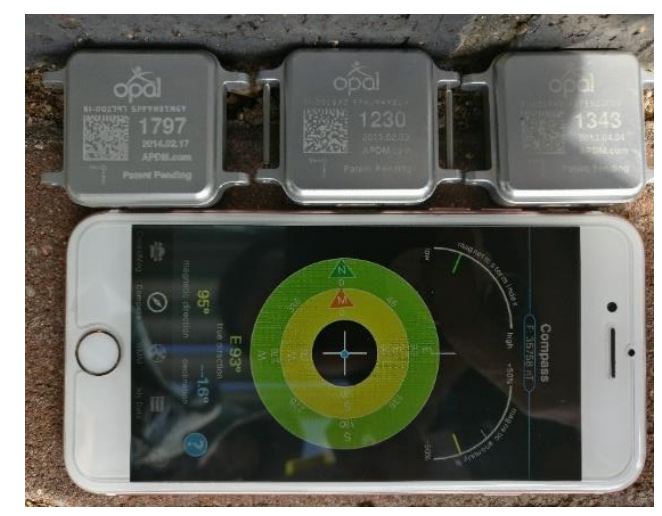

Figure 2. Inertial measurement unit Opal 
Table 1. Performance characteristics of Opal.

\begin{tabular}{cccc}
\hline Property & Accelerometer & Gyroscope & Magnetometer \\
\hline Axes & 3 & 3 & 3 \\
Range & $\pm 2 \mathrm{~g}, \pm 16 \mathrm{~g}$ & $\pm 2000^{\circ} \mathrm{s}$ & $\pm 6 \mathrm{G}$ \\
Noise density & $128 \mu \mathrm{g} / \sqrt{\mathrm{Hz}}$ & $0.07 \% / \mathrm{s} / \sqrt{\mathrm{Hz}}$ & $4 \mathrm{mG} / \sqrt{\mathrm{Hz}}$ \\
Max Sample rate & $1280 \mathrm{~Hz}$ & $1280 \mathrm{~Hz}$ & $1280 \mathrm{~Hz}$ \\
Bandwidth & $50 \mathrm{~Hz}$ & $50 \mathrm{~Hz}$ & $32.5 \mathrm{~Hz}$ \\
Resolution & $14 \mathrm{bits}$ & $14 \mathrm{bits}$ & $14 \mathrm{bits}$ \\
\hline
\end{tabular}

\section{Human actuator calibration and GRF reconstruction}

From Eq. (1) or (2), the GRF (i.e., the exciting force) of human activities can be reconstructed. To identify the appropriate feature point representing COM acceleration in the single rigid model, the calibration work should be done before the dynamic test.

In this section, human lateral swaying was first chosen as an example to show in detail the procedure of human actuator calibration and the GRF reconstruction. The calibration of the jumping force was then briefly discussed.

\subsection{Calibration test of swaying activity}

For most buildings, lateral sway is the dominant mode of vibration. To generate a lateral force from human activities, swaying is a good choice. It is also a common crowd activity during concerts, celebrations during sport games, and a popular group aerobic exercises in the gym (Lee et al., 2013). The calibration test was conducted in the gait laboratory of Shuguang hospital in Shanghai. In order to find out the most appropriate feature point representing the COM movement and reconstructing the GRF, six feature points, including the forehead, seventh cervical (C7) vertebra, sternum, lower back, navel, and right foot were chosen for the trial of GRF reconstruction. A set of four high-precision triaxial force plates were used to let testers sway without any restrictions, while measuring the force directly (shown in Figure 3). 


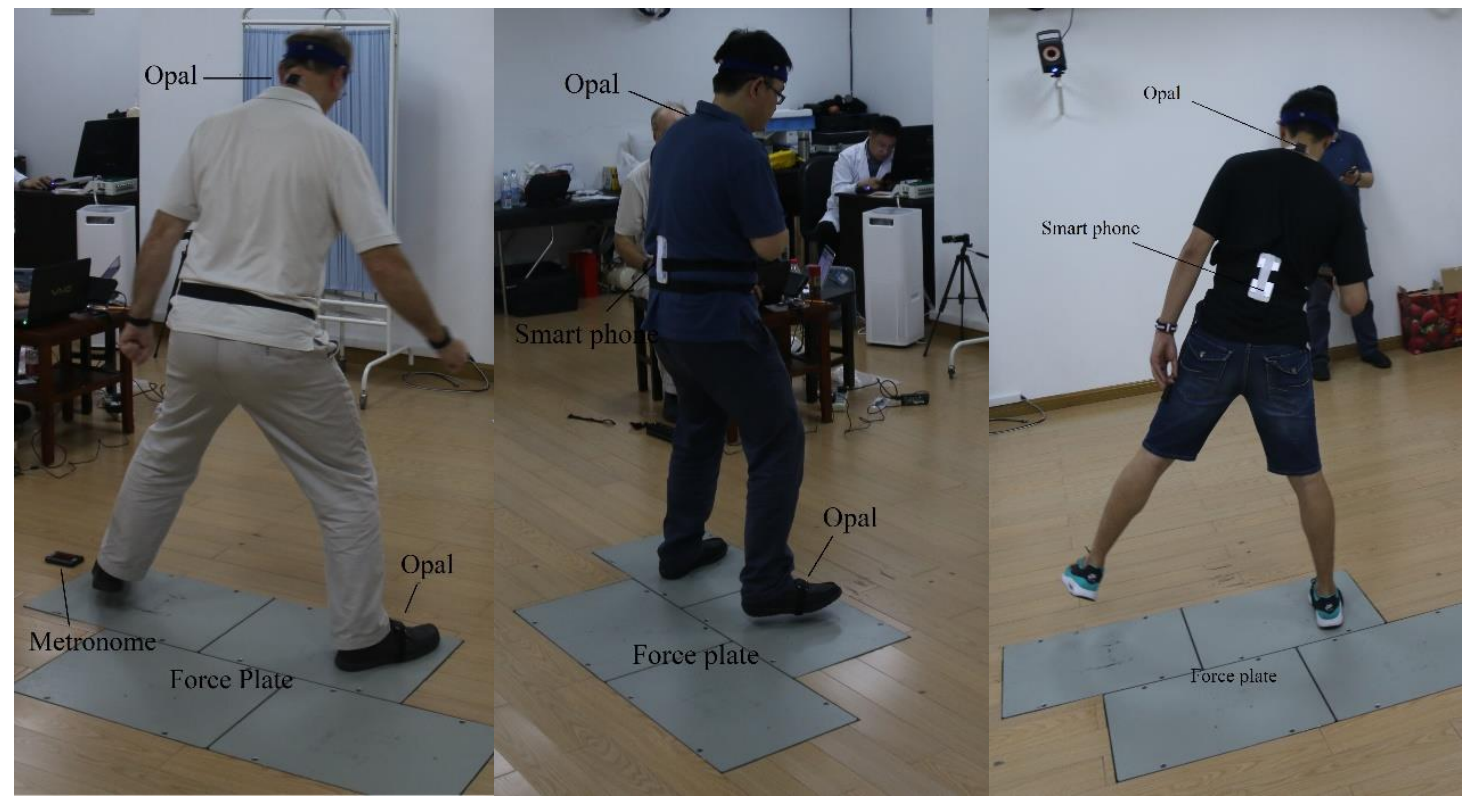

Figure 3. Calibration of human actuator in the gait lab (Tester A, B \& C at different swaying frequencies).

Three testers A, B, and C participated in the test, with ages of $50 \mathrm{y}^{+}, 40 \mathrm{y}^{+}$, and $20 \mathrm{y}^{+}$, heights of $185 \mathrm{~cm}, 175 \mathrm{~cm}$, and $178 \mathrm{~cm}$, and body weight of $89 \mathrm{~kg}, 85 \mathrm{~kg}$, and $66 \mathrm{~kg}$, respectively. All of the testers are researchers (professors and students) of this research project. Each tester swayed at various frequencies from $0.25 \mathrm{~Hz}-2.0 \mathrm{~Hz}(30-240 \mathrm{steps} / \mathrm{min})$ on the force plates. When swaying at a lower frequency, testers stand firmly at the middle of each cycle, raise their feet together, and sway their trunk widely to the other side simultaneously; this is similar to the side-by-side stamping in sumo wrestling. When the swaying frequency increases, testers have to lift and lower feet faster, reducing their trunk movement to keep up with metronome rhythm. This phenomenon clearly indicates a difference from the constant stroke motion of a mechanical shaker and is accompanied by a change of mass participant ratio with swaying frequency. The measured peak and root-mean-square (RMS) values of GRF shown in Figure 4 are consistent with these observations. 


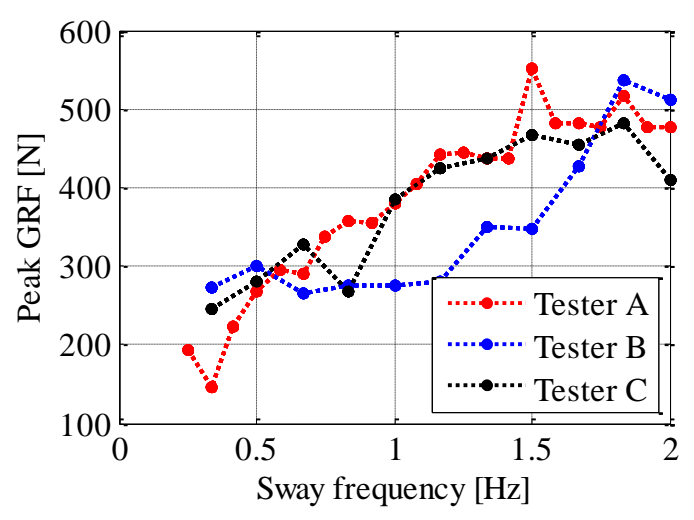

(a) Peak value

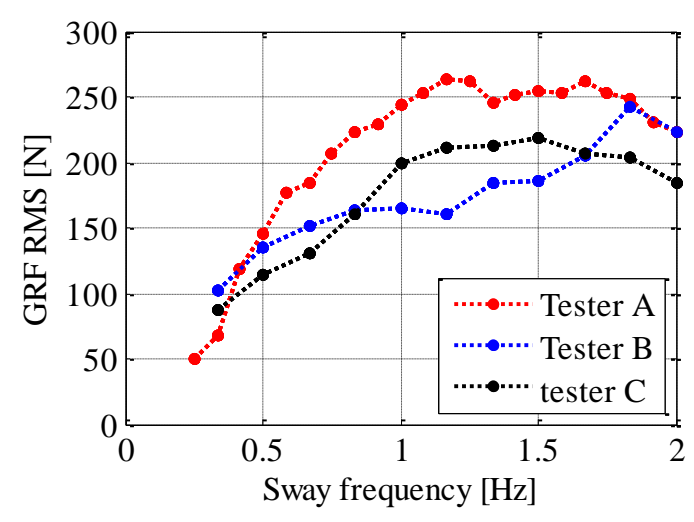

(b) Root mean square value

Figure 4. Variation of GRF with swaying frequency.

Figure 4 displays the variation of the peak GRF value and root-mean-square (RMS) value with the swaying frequency, showing a clear increasing trend. The reason is for higher swaying frequency, testers need a larger counterforce to help them change the swaying direction rapidly, but subject to human body capability, this trend slows down and tends to be constant when the tester sways faster than $1 \mathrm{~Hz}$.

Figure 5 shows how the RMS value of the lateral acceleration records at each feature point (measured by Opal) change with the increase of swaying frequency.
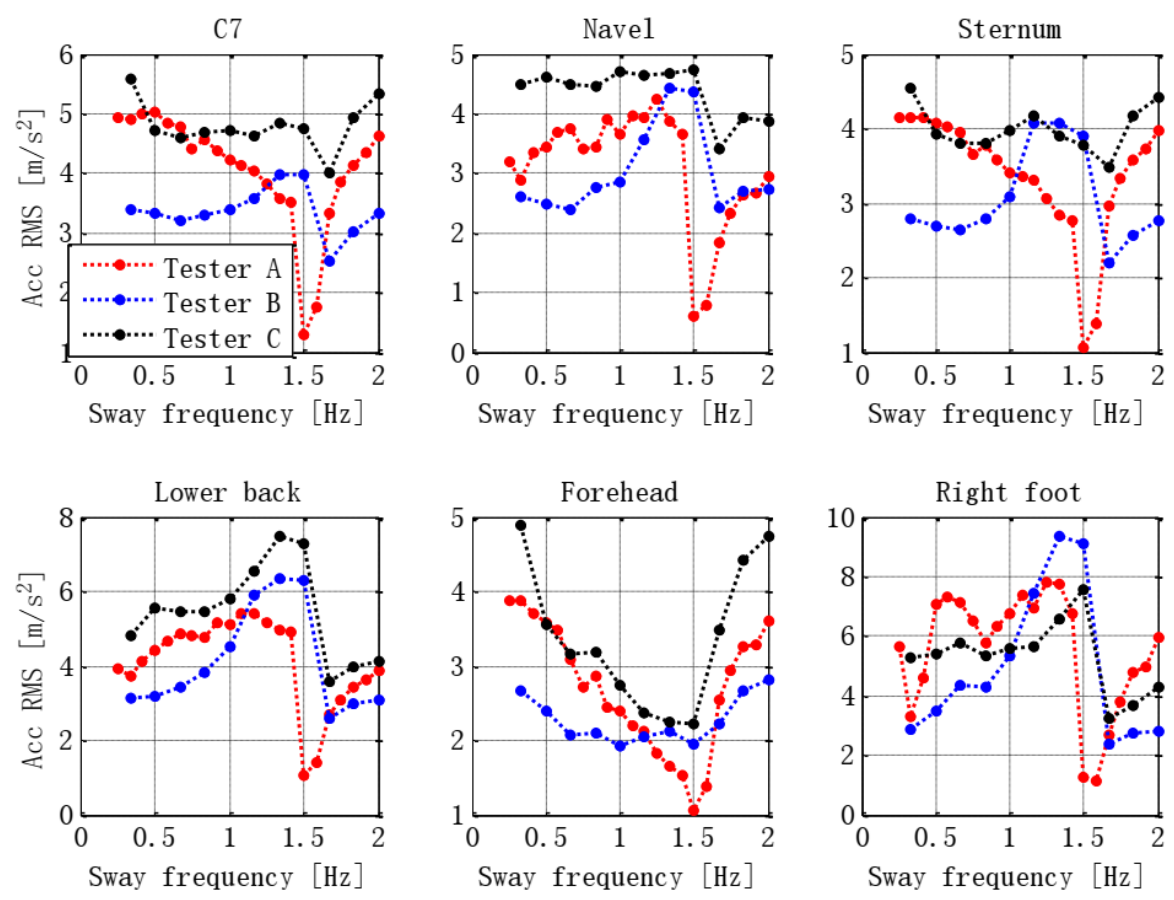

Figure 5. RMS accelerations of each feature point. 
Unlike the GRF, there is not such a clear relation between lateral acceleration and swaying frequency. For those six feature points, lateral accelerations maintain a higher value at lower frequency, coming to a steep decrease at around $1.5 \mathrm{~Hz}$ and rapid increase at higher frequency. A rapid change at around $1.5 \mathrm{~Hz}$ may be because it is the most comfortable swaying frequency for a tester, thus requiring the lowest force (i.e., acceleration).

\subsection{Mass participant ratio}

The mass participant ratio $R$ can be determined from Eq. (2) by introducing measured GRF (by force plates) and acceleration (by IMU) to both sides. As both GRF and acceleration are time histories with certain duration, the following equation is recommended to obtain a stable $R$ from zero-mean RMS values of the two records.

$$
R=\frac{\sqrt{\frac{1}{n_{1}} \sum_{i=1}^{n_{1}}\left(F_{i}-\bar{F}\right)^{2}}}{M \cdot \sqrt{\frac{1}{n_{2}} \sum_{j=1}^{n_{2}}\left(a_{j}-\bar{a}\right)^{2}}}
$$

where $M$ is the total mass of human body, $F_{i}$ and $\bar{F}$ are the measured time history of the GRF and its mean value, $a_{j}$ and $\bar{a}$ are measured feature point acceleration time history and its mean value, respectively, and $n_{1}$ and $n_{2}$ are the steady-state length of each time history (with sample frequency of $100 \mathrm{~Hz}$ ). In this study, the duration of each record is more than $30 \mathrm{~s}$. Figure 6 shows how the obtained mass participant ratio changes with the swaying frequency at different feature points:
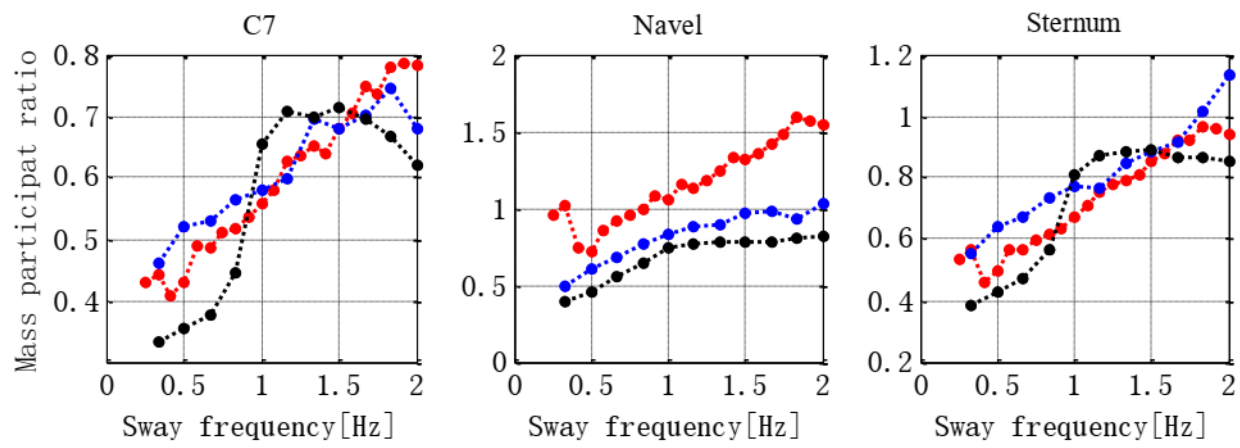

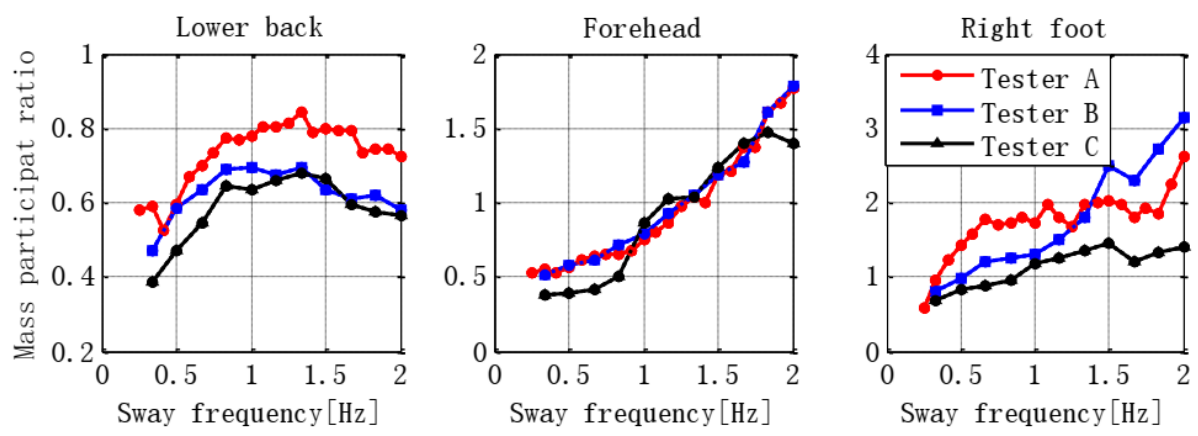

Figure 6. Mass participant ratio of each feature point.

Figure 6 shows a clearer linearly increasing trend between the mass participant ratio and swaying frequency for most feature points. It indicates that with the increase of swaying frequency, a larger proportion of the body mass contributes to the activity, which is consistent with the observed phenomena during the experiment. It also worth noting that Eq. (3) does not require that the timing of the force and acceleration be strictly synchronized, which greatly facilitates the calibration test.

The mass participant ratios for three testers are slightly different for the same swaying frequency, so it is better to calibrate the tester before performing any field test. Nevertheless, for the scenario of a rapid on-site preliminary test, an initial guess of $\mathrm{R}$ can be obtained from the linear fit of Figure 6 with $R^{2}$ value of 0.8122 , which is given in Eq. (4) and depicted in Figure 7.

$$
R_{\text {sway }}=0.211 f_{\text {sway }}+0.353
$$

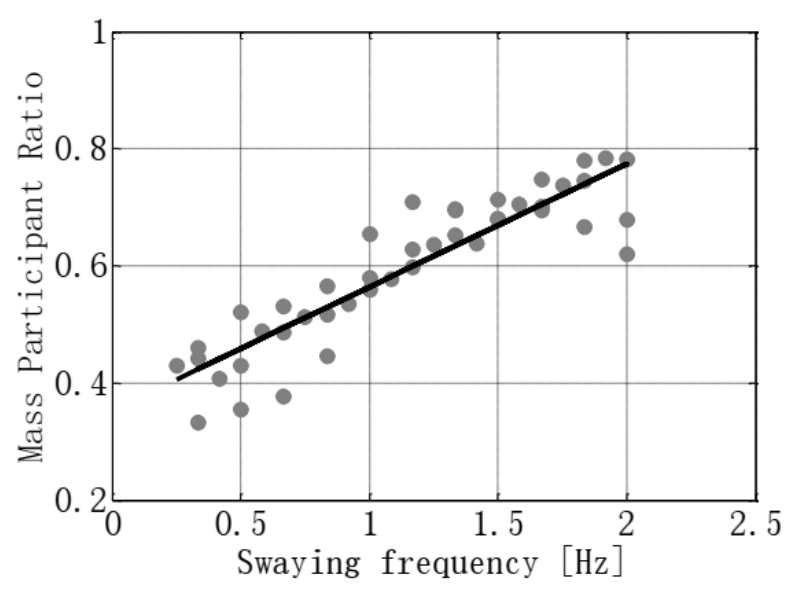


Figure 7 Linear fit of mass participant ratio with swaying frequency (C7).

With the calculated mass participant ratio, the GRF can be reconstructed using Eq. (2). An example of tester A swaying at $1.0 \mathrm{~Hz}$ is shown in Figure 8. It is clear that with the appropriate selection of the feature point, the reconstructed GRF fits the measured time history quite well, with a negligible error in the peak acceleration and curve shape indicating that the proposed method is suitable for GRF reconstruction.
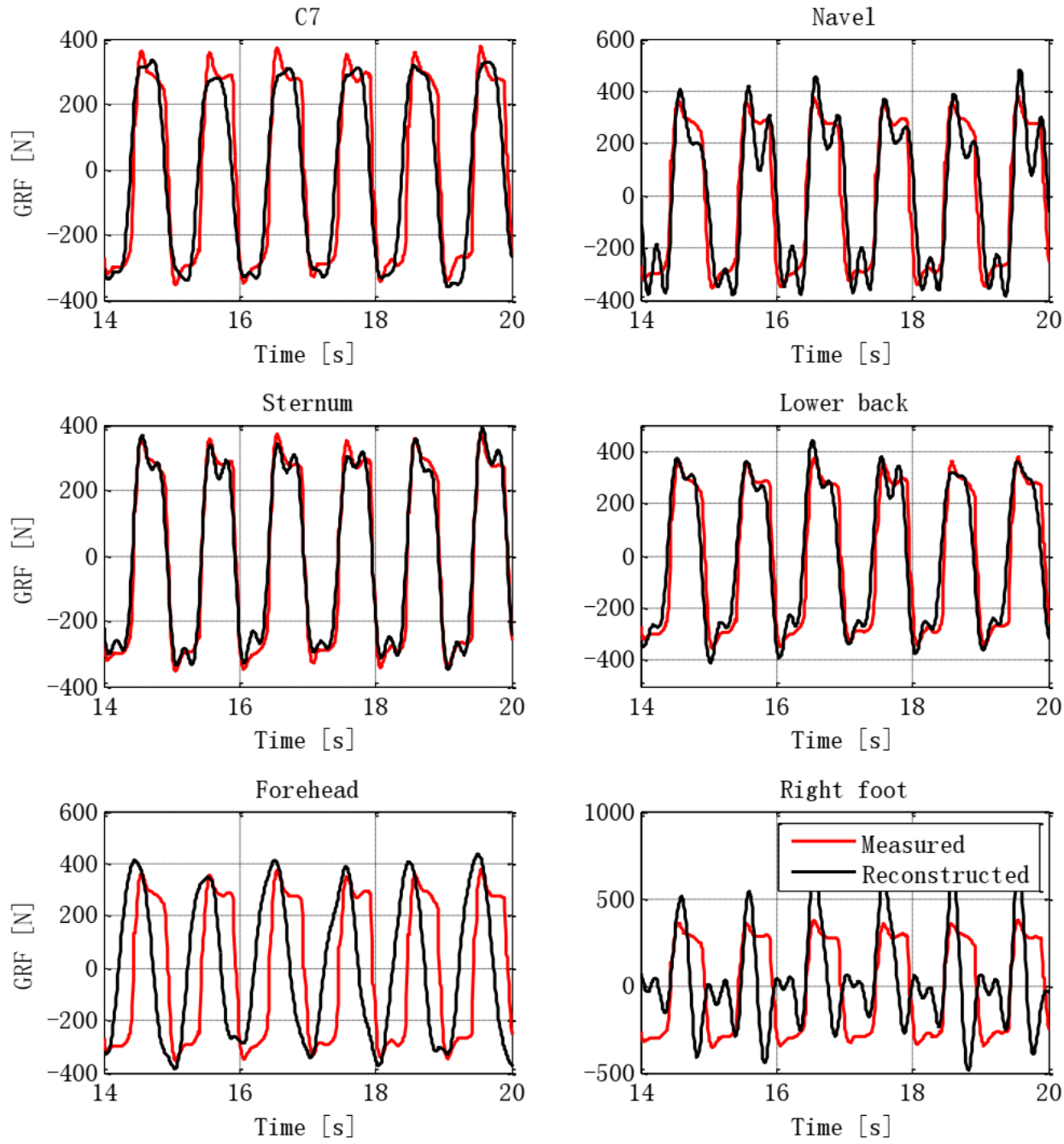

Figure 8. Comparison between measured and reconstructed GRF.

\subsection{Feature point for GRF reconstruction}

A comparison between the measured and reconstructed GRF in Figure 8 indicates that with 
the exception of the right foot, any feature point can be used to reconstruct the swaying force with acceptable accuracy. Mainly because of these points, especially the forehead, sternum, and C7 vertebra are located close to the body central line, and have little muscle or fat coverage, the data from these points are clear and steady.

Among all those applicable feature points, the $\mathrm{C} 7$ vertebra is easily located visually and by touch, with there being a small possibility of error in sensor fastening, also reducing the effects of the head movement (in the procedure) compared to the use of the forehead as a feature point. Furthermore, C7 is a widely used feature point in studies of human activities such as jumping or bouncing (Zhang et al., $2013 \&$ 2016, Chen et al., 2016); thus, it is recommended for the force reconstruction of human actuators.

\subsection{Consistency test of participant ratio}

To further verify the consistency of human actuators, a similar calibration experiment with tester C was later implemented in St Luke's biomechanical gait laboratory of the University of Exeter, as shown in Figure 9.

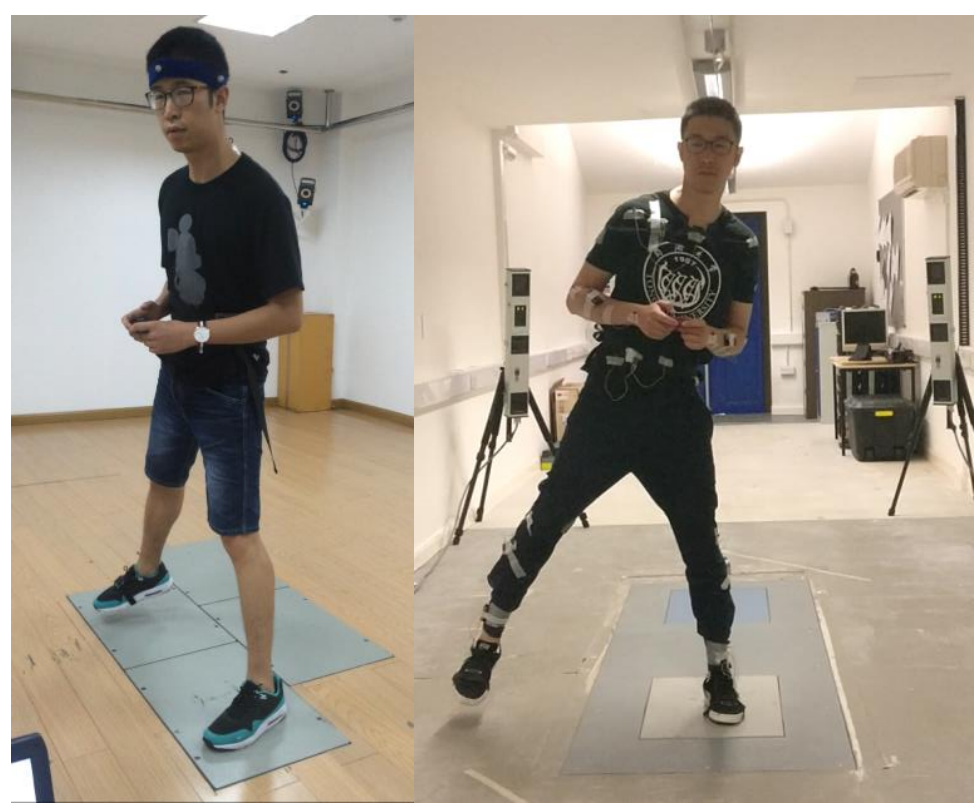

Figure 9. Repeated calibration of tester C in Shuguang hospital (left) and St Luke's (right).

For the limitation of laboratory conditions, the single force plate in St Luke's laboratory 
was only able to measure GRF of one foot at a time. To calculate the mass participant ratio, the human body and swaying force were assumed to be perfectly symmetrical, so the obtained mass participant ratio of the $\mathrm{C} 7$ vertebra compared with the former ones are as shown below.

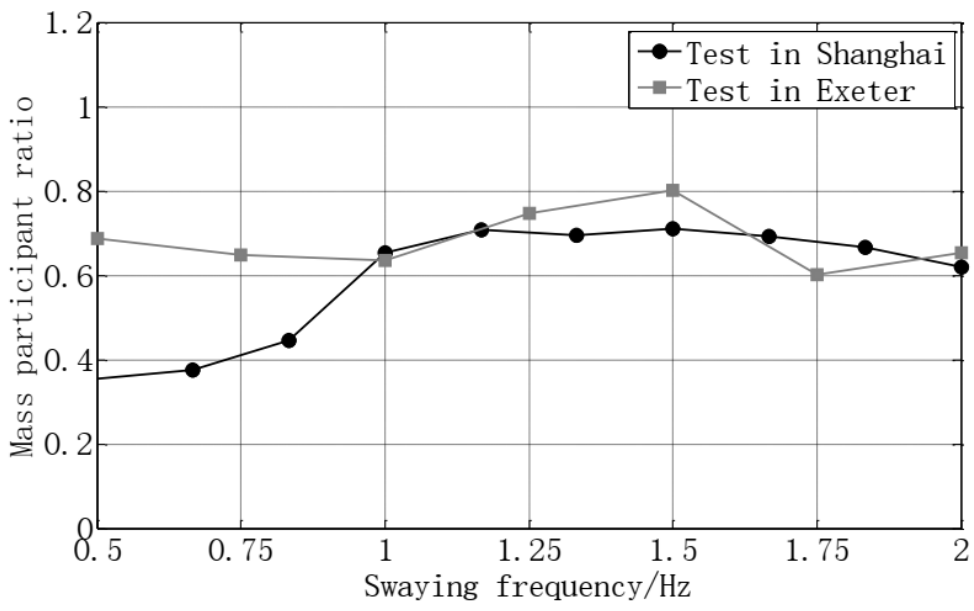

Figure 10. Comparison of two calibration experiments for $\mathrm{C} 7$ vertebra.

Figure 10 shows that $\mathrm{R}$ values in the higher frequency zone $(>1 \mathrm{~Hz})$ agree well but have obvious difference for lower frequencies. The body weight increase $(4 \mathrm{~kg})$ of tester B between to tests is not strong enough explain this phenomenon, thus the force analysis graph during low sway frequency is given in following Figure 11.

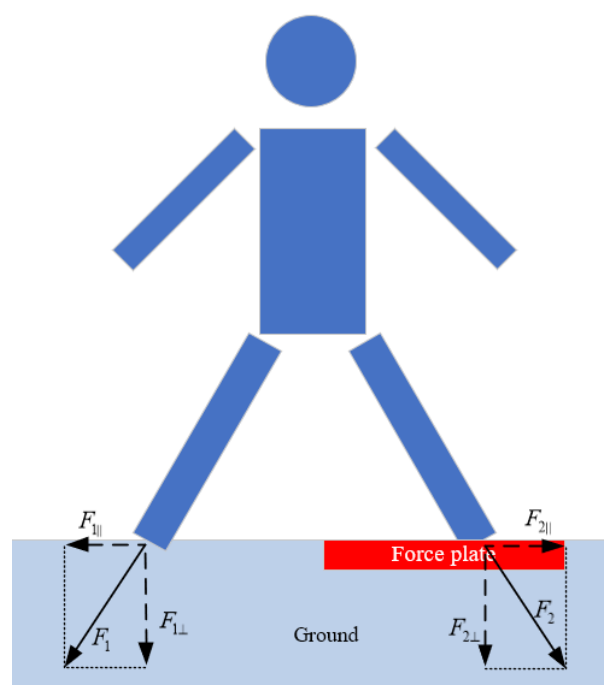

Figure 11. Force analysis during low frequency swaying.

When swaying frequency is low, tester has one foot kept contact with ground when the other foot step on the force plate to maintain the body balance, generate two forces $F_{1}$ and 
$F_{2}$ as shown in Figure 11, each can be decomposed as $F_{\|}$and $F_{\perp}$. The true lateral GRF should be $\left(F_{2 \mid}-F_{1 \|}\right)$, but the single force plate can only measure the $F_{2 \mid}$, which is obvious larger, makes the calculated mass participant ratio larger than the results in previous test.

In general, the tester's mass participant ratio is acceptably consistent between two calibration tests, but if there is a non-negligible body condition change, the calibration data need to be updated.

\subsection{Calibration of jumping activity}

Jumping activity can be used to generate a vertical force that drives a vertical response of a structure. The calibration procedure similar to that of swaying was applied to a tester D (age $20 \mathrm{y}+$, height $190 \mathrm{~cm}$, body weight $96 \mathrm{~kg}$ ) to determine the mass participant ratio of the jumping activity. This time, the $\mathrm{C} 7$ vertebra was directly chosen as the feature point, and its acceleration measured by Opal was adopted for excitation reconstruction. Six jumping frequencies were tested. For each case, the mass participant ratio was calculated using Eq. (3).

Figure 12 shows the variation of the mass participation ratio with jumping frequency; a linear fit (Eq. 5) with $R^{2}$ value of 0.9742 can represent the relationship well.

$$
R_{\text {jump }}=-0.077 f_{\text {jump }}+1.131
$$

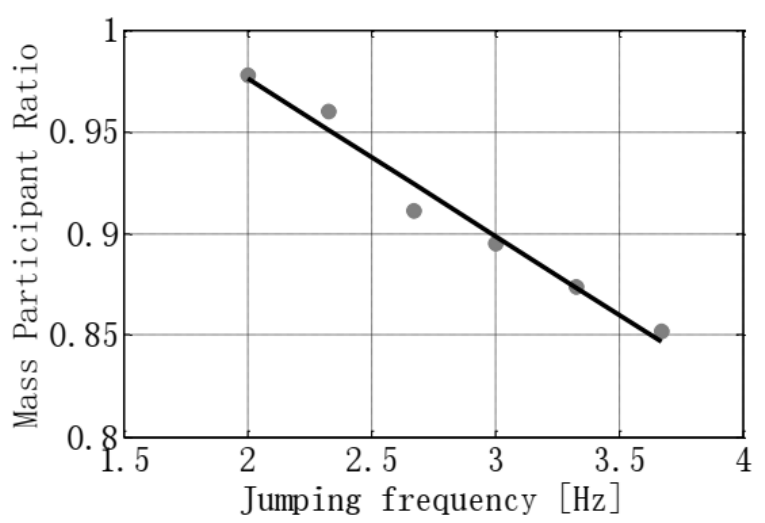

Figure 12. Mass participant ratio of jumping load. 


\section{Application}

The proposed human actuator testing approach was applied to two structures: an eightstory steel frame building and a long-span floor. Dynamic properties in terms of natural frequencies, such as the damping ratios and modal masses, were determined and compared with those obtained by more traditional test methods and numerical analyses.

\subsection{Case 1: 8-story steel frame building}

\subsubsection{Test building}

In order to verify the feasibility of the proposed dynamic test technology, field tests were conducted on a typical office building on the campus of Tongji University. The office building (ichnography in Figure 13) combines two separate steel frame constructions connected by a three-story footbridge. The length, width, and height of the measured part (zone I) is $67 \mathrm{~m}, 16$ $\mathrm{m}$, and $32.875 \mathrm{~m}$, respectively, containing 8 floors above ground and 1 floor basement. The foundations of both buildings are made of reinforced concrete and are separate from each other. The above-ground part is the steel-braced frame structure, with a 135-mm-thick floor made of profiled steel plate and reinforced concrete.

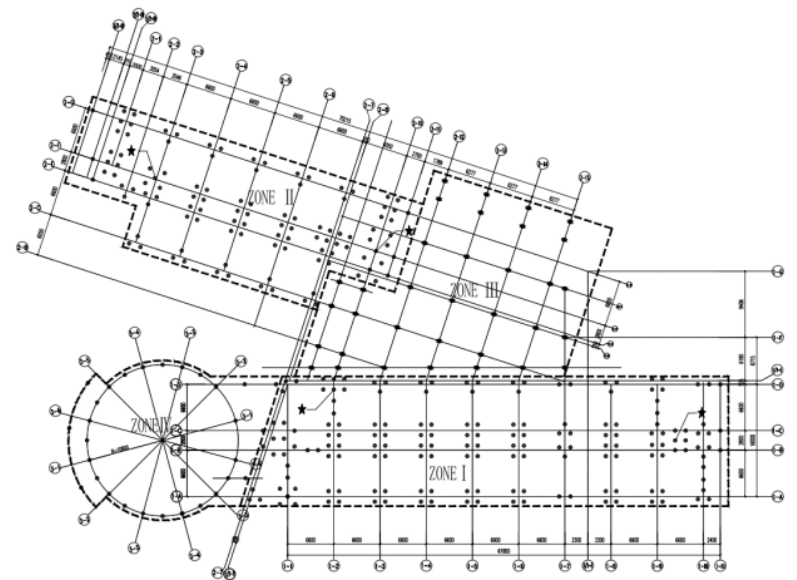

Figure 13. Ichnography of tested building (zone I). 
The natural frequency of the first lateral mode of vibration was calculated as $1.505 \mathrm{~Hz}$ with a corresponding modal mass of 4383 metric ton (normalized to unity maximum horizontal component of mode shape).

\subsubsection{Test procedure}

To conduct modal testing, previously calibrated testers A and B swayed individually and synchronously on the top floor of the building, and the test was implemented using the following procedures:

(1) Opal sensors were used to measure the modal frequency of the structure by ambient vibration testing. Spectra of the acceleration response were calculated, and the natural frequency $f_{L}$ of the first lateral vibration model was estimated by peak-picking.

(2) Testers swayed at the middle of the top floor following the guidance of a metronome whose beats were adjusted to the measured natural frequency $f_{L}$, attempting to generate the resonance response. The testers' lateral accelerations and structural responses were then recorded using wearable sensors attached to their C7 vertebra and corridor's side wall.

(3) Testers excited the structure for more than $30 \mathrm{~s}$, then stopped suddenly and kept measuring the free decay of the structural vibration.

\subsubsection{Test results}

Figure 14 demonstrates that swaying at the selected frequency, testers can excite obvious resonant response, and a build-up procedure can be observed. With one tester $(89 \mathrm{~kg})$ swaying, the structural peak acceleration response reached $2 \mathrm{~mm} / \mathrm{s}^{2}$, while with two testers swaying together (total mass $174 \mathrm{~kg}$ ), the structural peak response reached $3 \mathrm{~mm} / \mathrm{s}^{2}$. 


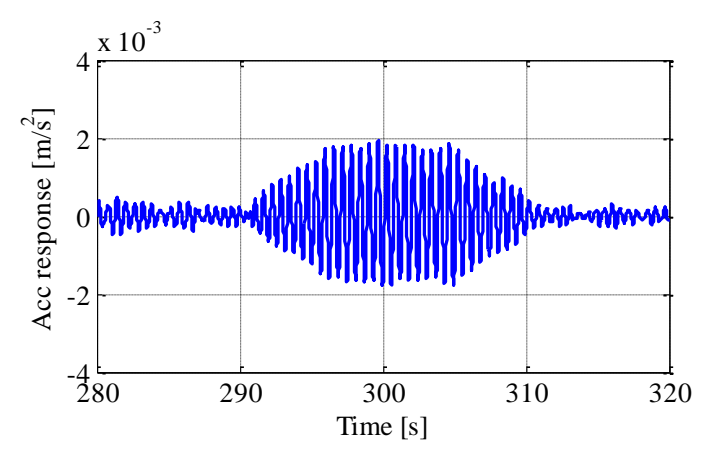

(a) Vibration generated by one tester

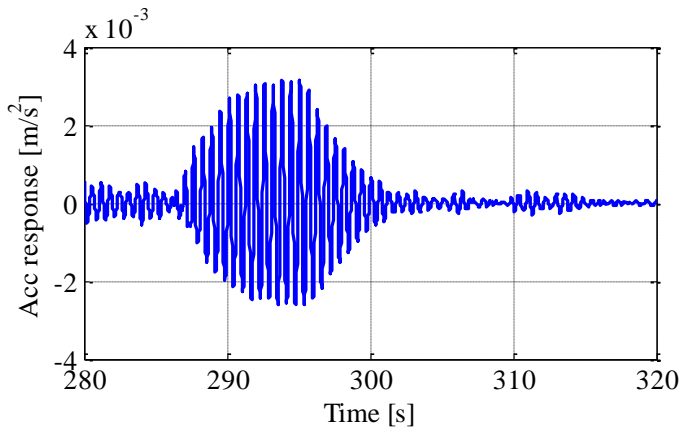

(b) Vibration generated by two testers

Figure 14. Structural response under human excitation.

\subsubsection{Modal parameters}

The structural natural frequency estimated from the ambient vibration test was $1.625 \mathrm{~Hz}$, in the lateral sway mode, very close to results measured in Shi et al. (2012) using traditional accelerometers. However, these experimental frequency estimates are larger than the fundamental mode frequency estimate of $1.505 \mathrm{~Hz}$ from numerical (finite element) analyses that ignored the effects of filler walls and floor slabs. Such large differences between predicted and measured frequencies emphasize the necessity for field tests.

From the structural free decay curve, the modal damping ratio can be identified by following Eq. 6:

$$
\delta=\ln \frac{A_{i}}{A_{i+1}}=\frac{2 \pi \zeta}{\sqrt{1-\zeta^{2}}}
$$

In which $\delta$ is so-called logarithmic decrement of the free decay vibration, $A_{i}$ and $A_{i+1}$ are successive peaks of decay time history, $\zeta$ is the wanted damping ratio. From time history shown in Figure 14, $\zeta$ can be easily calculated, which was $2.58 \%$.

The swaying force is necessary to identify the modal mass. In this case, the mass of tested building is obvious great larger than human tester (over 1000 times), thus the interaction between human and structure (HSI) (Jimenez-alonso et al., 2016, Nimmen et al., 2017) is ignored. By assuming the GRF is exactly same as it was on rigid floor, the mass participant ratio of tester A at a swaying frequency of $1.625 \mathrm{~Hz}$ is determined as 0.727 based on calibration 
results shown in Figure 6. Furthermore, with the measured $\mathrm{C} 7$ vertebra acceleration, the GRF can be reconstructed using Eq. (2), shown in Figure 15.

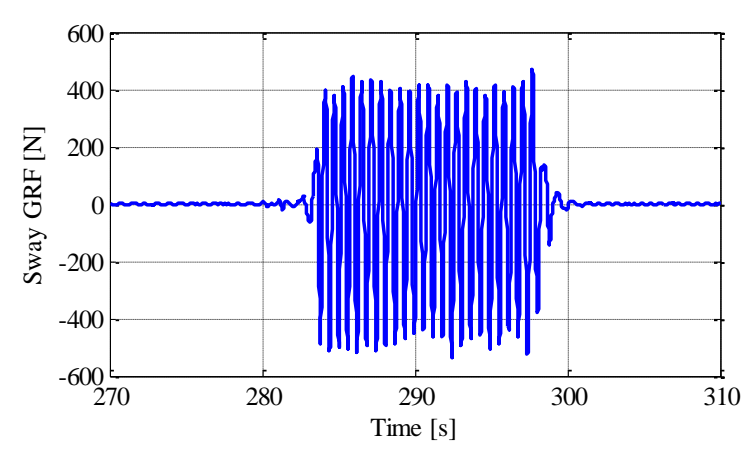

Figure 15. Lateral GRF reconstructed by tester A's C7 acceleration.

The human actuator aims to generate a resonant response of the structure to the forcing. We can therefore assume the building behaves as a SDOF system. With the reconstructed excitation input, the measured natural frequency, damping ratio, and assumed mass, the acceleration responses of the SDOF system can be easily calculated numerically. For modal mass estimation, a simple equation with perfectly sinusoidal excitation at the exact natural frequency for long enough to reach steady state and SDOF assumption is commonly used, shown in following equation (7):

$$
m=F_{\text {peak }} /\left(2 \zeta a_{\text {peak }}\right)
$$

In which $m$ is modal mass, $F_{\text {peak }}$ and $a_{\text {peak }}$ are peak value of excitation force and structural response, $\zeta$ is structural damping ratio.

According to equation (7), the modal mass of tested structure is 5631 metric ton. However, as the excitation force generated by human actuator is not so stable and regular and is highly sensitive to small errors in the damping estimation -which can be greater for shorter decays, a time history analysis method was finally selected. By adjusting the assumed modal mass continuously and comparing the numerical and measured acceleration responses, the appropriate modal mass can be identified. The Newmark- $\beta$ method was used in this research. By taking the steady-state RMS value of the acceleration response as the indicator, the modal mass was estimated as 4530 metric ton (Figure 16), which is only $3.4 \%$ different from the 
numerical analysis estimate, providing a limited validation of the method.

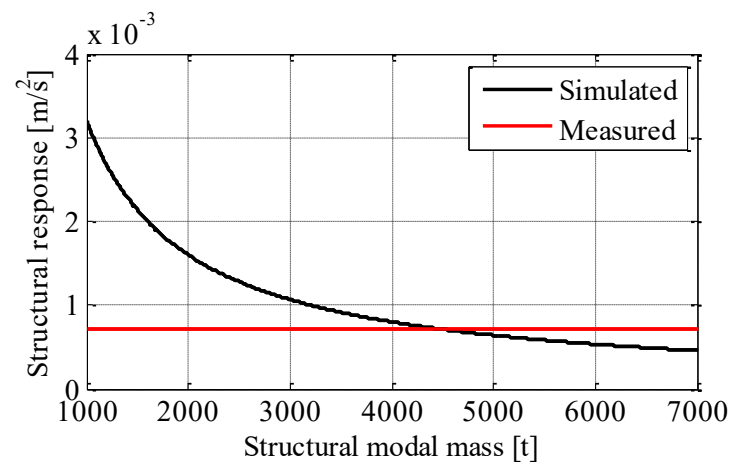

Figure 16. Modal mass estimation.

\subsection{Case 2: Long-span floor}

\subsubsection{Test floor}

For the field test with human vertical excitation, a $60 \mathrm{~m} \times 36 \mathrm{~m}$ large span concrete floor was chosen. The floor was divided into $5 \times 3$ panel of $12 \mathrm{~m} \times 12 \mathrm{~m}$ panels by columns and girders, and the test was held on the middle panel of the floor, as shown in Figure 17.
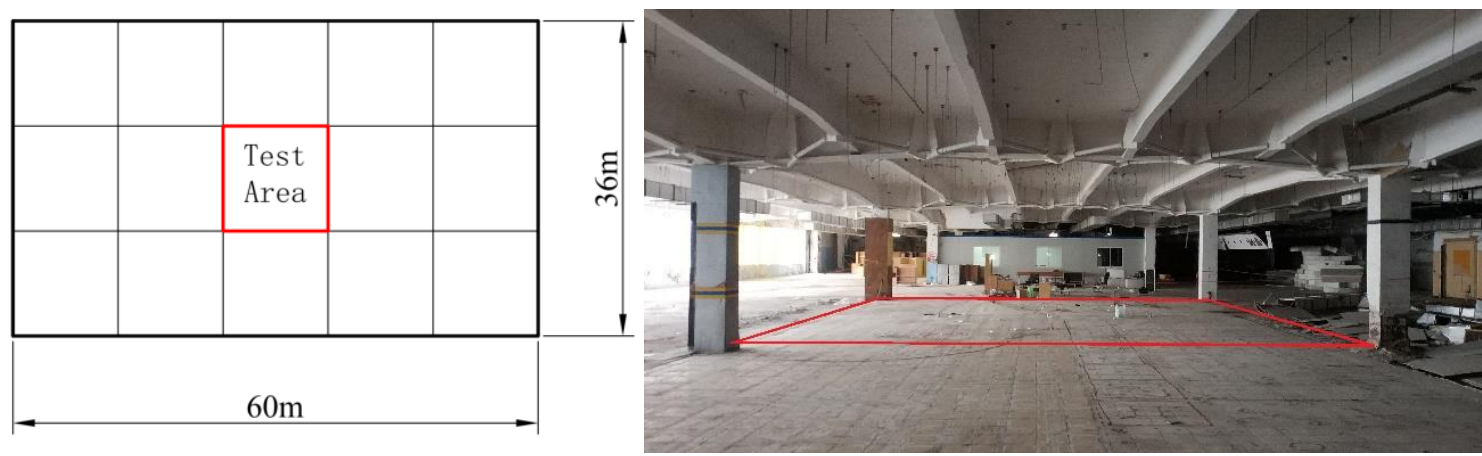

Figure 17. Field test site of the floor (red squared part).

\subsubsection{Test procedure}

For comparison purposes, the ambient vibration test and hammer test were first conducted to estimate the natural frequencies and modal masses of the floor. The results are given in Table 2. The modal mass was estimated using hammer test data by the eigensystem realization algorithm (ERA), which is integrated in the data acquisition system and normalization to unity maximum mode shape ordinate is chosen by default. 
Table 2. Dynamic properties of the floor.

\begin{tabular}{cccc}
\hline No. & Frequency [Hz] & Damping ratio [\%] & Modal mass [t] \\
\hline 1 & 5.35 & 1.57 & 8.73 \\
2 & 8.17 & 2.04 & 28.89 \\
3 & 10.53 & 1.44 & 4.96 \\
4 & 16.63 & 0.90 & 7.85 \\
\hline
\end{tabular}

On this floor, the tester D (whose jumping force was calibrated in section 4.4) jumped at $2.68 \mathrm{~Hz}$, which is half of the frequency of the first vertical mode. In this case, although the structure's vibration is strong and easy to perceive by surrounding people, the exciter himself could hardly feel the vibration, thus the jumping force could be assumed as the same on a rigid floor. Similar assumption was used by other researchers when studying HSI of human-induced loads (Nimmen et al., 2017; Shahabpoor et al., 2017). The corresponding mass participant ratio $\mathrm{R}$ for this frequency is determined from the calibration result (Figure 11) as 0.911.

\subsubsection{Model parameters}

Figure 18 shows the jumping force reconstructed by feature point acceleration and corresponding floor response during the test.
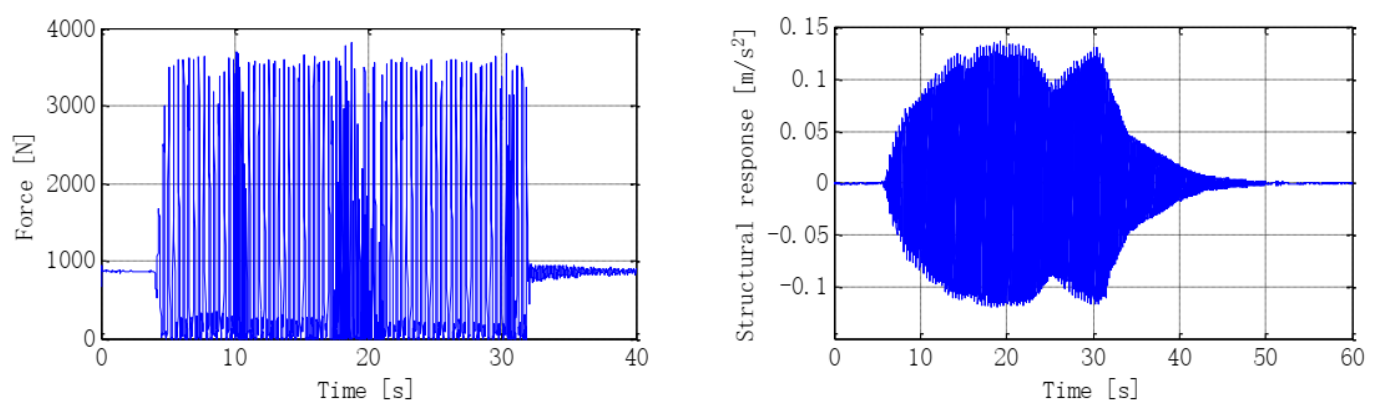

Figure 18. Jumping excitation and corresponding structural response.

With a single tester $\mathrm{D}$ of weight $96 \mathrm{~kg}$ jumping at $2.68 \mathrm{~Hz}$ on the center of the testing zone, the floor peak acceleration response increased to $0.14 \mathrm{~m} / \mathrm{s}^{2}$. From the free decay part of the structural response, the floor damping ratio of the first mode was estimated as $0.59 \%$.

Using the same procedure given in section 5.1.3, the modal mass identification procedure 
is shown in Figure 19; the estimated mass of the floor is 18.03 metric ton, which is not so close to the result obtained with the impact hammer test shown in Table 2, mainly because the tested floor is quite large, impact hammer may not able to excite whole panel, not mention the nearby panels are connected with the tested one, under continuous human excitation, the tested panel reach the steady state vibration, the panel is fully excited thus the result is more convincible.

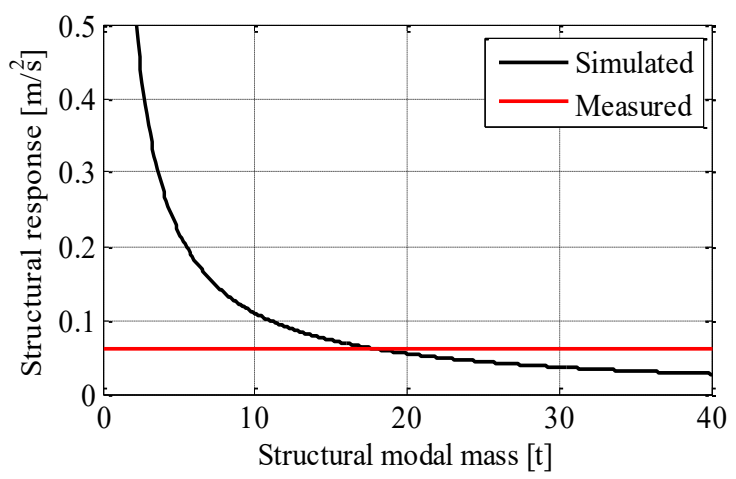

Figure 19. Floor modal mass estimation.

Furthermore, from designer's view, it can be assumed that Young's modulus $E$ of the concrete floor is $30 \mathrm{kN} / \mathrm{mm}^{2}$, Poisson's ratio $v$ is 0.2 , plate equivalent thickness $t$ is $0.24 \mathrm{~m}$, density of concrete is $25 \mathrm{kN} / \mathrm{m}^{3}$. For simply supported plate on four sides, its fundamental frequency and modal mass can be calculated as following equation (8) and (9) (Hechler et al., 2008):

$$
\begin{gathered}
f=\frac{\alpha}{l^{2}} \sqrt{\frac{E t^{3}}{12 m\left(1-v^{2}\right)}} \\
\alpha=1.57\left(1+\lambda^{2}\right), \quad \lambda=l / b \\
M_{\text {mod }}=\beta \cdot M_{t o t}
\end{gathered}
$$

In which $l$ and $b$ are the length and width of the floor, $m$ is areal density of the floor (including its own weight), $M_{\text {mod }}$ and $M_{\text {tot }}$ are modal mass and total mass of the structure, for any $\lambda$, factor $\beta$ approximately equals to 0.25 .

With equation (8), (9) and assumptions above, the floor's fundamental frequency is 5.34 $\mathrm{Hz}$ and modal mass is 21.6 metric ton, both close to human actuator test results. In contrast, the results obtained by hammer test are not so reliable. There are signal processing issues relating 
to hammer testing, such as windowing, and the signal to noise ratio can be poor, since energy input with mechanical and human actuators is usually many orders of magnitude greater than with hammer impulse excitation. In fact, hammer test is not so recommended when high quality modal test data is required (Reynolds P \& Pavic A., 2000).

\section{Discussion}

Although structural dynamic characteristics of existing structures are very important, commonly used technologies for modal testing have disadvantages with respect to the logistical requirements for equipment transportation and installation, electric power requirements, complex operating procedures and high costs, making them practical only for a limited number of structures. A simplified dynamic testing technology using a human actuator is proposed in this study, and offers a possible solution to the problem. Human actuators are of course much easier to transport, have no need for complex signal generators (except for a metronome), for installation fixtures, or for electric power supplies. The authors have already integrated the data acquisition and parameter identification functions into a cellphone application, with which a single person carrying two wearable sensors would be able to conduct quick structure modal testing at low cost, producing estimates of mode frequency, damping, and mass with acceptable accuracy.

Although it is convenient and easy to apply, the proposed human actuator test technology still has many limitations:

(1) The feature points (for IMU attachment) cannot perfectly represent the movement of the body barycenter, leading to deviations in exciting force reconstruction. The accuracy of the dynamic testing depends strongly on the accuracy of the calibration.

(2) The dynamic testing is based on the resonant response, making the technology capable primarily of identifying specific mode characteristics one at a time. 
(3) As the mode shape of the structure is not directly measured but rather estimated by empirical formula or FE model (if full ambient testing is not possible, e.g., because of a limited number of sensors), finding the suitable location for actuator or knowing the correct modal ordinate for scaling to unity maximum mode shape ordinate may introduce errors.

\section{Conclusion}

This study shows that: the human excitation dynamic modal testing technology is suitable for quick field tests of regular, low-frequency structures. By performing in advance appropriate calibrations, the dynamic testing can be processed by any normal engineering staff carrying several wearable sensors. Some specific findings regarding human actuators are as follows.

(1) The human body single rigid model is suitable for reconstructing swaying and jumping lateral forces. Owing to differences between testers (height, weight, age, etc.) the mass participant ratio of each person is slightly different. When applied in practical tests, the human actuator must be pre-calibrated. The ratio given in this research is only available as a reference.

(2) For the feature point selection of swaying excitation, the forehead, C7 vertebra, and sternum performed better compared to the navel, lower back, and right foot. For practical use in actual dynamic testing, as well as the need for vertical excitation, the C7 vertebra is proposed for both swaying and jumping activities.

(3) Applications on a typical frame construction and a long span floor show the practicability and accuracy of the suggested technology. The identified structural response, damping ratio, and modal mass have acceptable accuracy for most applications (e.g. assessment, design or design of retrofit), while also the testing procedure is simple and easy to repeat. However, human athletic ability, the mode frequency (or its 2 times, $1 / 2$ times value) should be within the range usually $0.25-5.0 \mathrm{~Hz}$. 


\section{Acknowledgement}

The authors would like to acknowledge the financial support provided by National Natural Science Foundation of China (51778465) and the State Key Laboratory for Disaster Reduction of Civil Engineering (SLDRCE14-B-16).

\section{References}

[1] A Practical Test: Wembley 1923, Pathe, United Kingdom, 1923. http://www.britishpathe.com/video/a-practical-test-wembley.

[2] Allen D L. Vibrational behavior of long-span floor slabs[J]. Canadian Journal of Civil Engineering, 1974, 1(1): 108-115.

[3] Aggarwal J K, Cai Q. Human motion analysis: A review[J]. Computer vision and image understanding, 1999, 73(3): 428-440.

[4] Au S K. Fast Bayesian FFT method for ambient modal identification with separated modes[J]. Journal of Engineering Mechanics, 2011, 137(3): 214-226.

[5] Siu-Kui AU. Operational Modal Aanlysis: Modeling, Bayesian Inference, Uncertainty Laws [M]. SPRINGER, 2018.

[6] Blakeborough A, Williams M S. Measurement of floor vibrations using a heel drop test[J]. Proceedings of the Institution of Civil Engineers-Structures and Buildings, 2003, 156(4): 367-371.

[7] Bobbert M F, Schamhardt H C, Nigg B M. Calculation of vertical ground reaction force estimates during running from positional data[J]. Journal of biomechanics, 1991, 24(12): 1095-1105.

[8] Bocian M, Brownjohn J M W, Racic V, et al. A framework for experimental determination of localised vertical pedestrian forces on full-scale structures using wireless attitude and heading reference systems[J]. Journal of Sound and Vibration, 2016, 376: 217-243.

[9] Brownjohn J M W, Pavic A. Experimental methods for estimating modal mass in footbridges using human-induced dynamic excitation[J]. Engineering Structures, 2007, 29(11): 2833-2843. 
[10] Brownjohn J M W, Bocian M, Hester D, et al. Footbridge system identification using wireless inertial measurement units for force and response measurements[J]. Journal of Sound and Vibration, 2016, 384: 339-355.

[11] Brownjohn J M W, Reynolds P, Fok P. Vibration serviceability of Helix bridge, Singapore[J]. 2016.

[12] Brownjohn J M W, Chen J, Bocian M, et al. Using inertial measurement units to identify medio-lateral ground reaction forces due to walking and swaying[J]. Journal of Sound and Vibration, 2018, 426: 90-110.

[13] Canadian Standards Association (CSA). Steel structures for buildings-limit state design. Appendix G: Guide for floor vibrations[J]. CAN3-S16. 1-M84, 1994.

[14] Chen J, Xu Y L. Application of HHT for modal parameter identification to civil structures[J]. Journal of vibrarion engineering, 2003. (In Chinese)

[15] Chen J, Zhang M.S. and Liu W., Vibration serviceability performance of an externally prestressed concrete floor during daily use and under controlled human activities, J. of Performance of Constructed Facilities, 2015, 04015007-1

[16] Chen J, Tan H, Pan Z. Experimental validation of smartphones for measuring humaninduced loads[J]. Smart Structures \& Systems, 2016, 18(3): 625-642.

[17] Chen J. Human-induced load and structural vibration[M]. Science Press, 2016. (In Chinese)

[18] Cheng C K, Chen H H, Chen C S, et al. Segment inertial properties of Chinese adults determined from magnetic resonance imaging[J]. Clinical biomechanics, 2000, 15(8): $559-566$.

[19] Dallard P, Fitzpatrick T, Flint A, et al. London Millennium Bridge: pedestrian-induced lateral vibration[J]. Journal of Bridge Engineering, 2001, 6(6): 412-417.

[20] Doebling S W, Farrar C R, Prime M B, et al. Damage identification and health monitoring of structural and mechanical systems from changes in their vibration characteristics: a literature review[J]. 1996.

[21] Ewins D J. Modal testing: theory and practice[M]. Letchworth: Research studies press, 1984.

[22] Friswell M, Mottershead J E. Finite element model updating in structural dynamics[M]. Springer Science \& Business Media, 2013. 
[23] Feldmann M, Heinemeyer C, Lukic M. Human-induced vibration of steel structures (Hivoss) [J]. Office for Official Publications of the European Communities, Luxembourg, 2010.

[24] Glanville M J, Kwok K C, Denoon R, et al. Full-scale damping measurements of structures in Australia[J]. Journal of Wind Engineering and Industrial Aerodynamics, 1996: 349-364.

[25] Hechler O, Feldmann M, Heinemeyer C, et al. Design guide for floor vibrations [C]//Proceedings of EuroSteel 2008 Conference. 2008.

[26] Housner G W, Bergman L A, Caughey T K, et al. Structural control: past, present, and future[J]. Journal of engineering mechanics, 1997, 123(9): 897-971.

[27] Jimenez-alonso J F, Saez A, Caetano E, et al. Vertical Crowd-Structure Interaction Model to Analyze the Change of the Modal Properties of a Footbridge[J]. Journal of Bridge Engineering, 2016, 21(8).

[28] Lee S H, Lee K K, Woo S S, et al. Global vertical mode vibrations due to human group rhythmic movement in a 39 story building structure[J]. Engineering Structures, 2013, 57 : 296-305.

[29] Lenzen K H. Vibration of steel joist-concrete slab floors[J]. AISC. Eng. Jour., 1966, 3: 133-136.

[30] Murray T M, Allen D E, Ungar E E. Floor vibrations due to human activity[M]. Steel design guide 11. American Institute of Steel Construction, 2003.

[31] Van Nimmen K, Lombaert G, De Roeck G, et al. The impact of vertical human-structure interaction on the response of footbridges to pedestrian excitation[J]. Journal of Sound and Vibration, 2017: 104-121.

[32] Ohlsson S V. Dynamic characteristics of cable-stayed bridges-Nonlinearities and weakly coupled modes of vibration[C]//Proc. of the int. conf. on cable-stayed bridges. 1987: 421431.

[33] Okauchi I, Tanaka A, Iwaya K, et al. Vibration test of Ohnaruto Bridge to confirm windproofness[C]//IABSE symposium. 1986.

[34] Pavic A, Reynolds P. Experimental assessment of vibration serviceability of existing office floors under human-induced excitation[J]. Experimental Techniques, 1999, 23(5): 41-45. 
[35] Pearsall D J, Reid J G, Ross R. Inertial properties of the human trunk of males determined from magnetic resonance imaging[J]. Annals of biomedical engineering, 1994, 22(6): 692706.

[36] Peeters B, Van der Auweraer H, Vanhollebeke F, et al. Operational modal analysis for estimating the dynamic properties of a stadium structure during a football game[J]. Shock and Vibration, 2007, 14(4): 283-303.

[37] Racic V, Brownjohn J M W, Pavic A. Reproduction and application of human bouncing and jumping forces from visual marker data[J]. Journal of Sound and Vibration, 2010, 329(16): 3397-3416.

[38] Racic V, Pavic A, Brownjohn J M W. Modern facilities for experimental measurement of dynamic loads induced by humans: A literature review[J]. Shock and Vibration, 2013, 20(1): 53-67.

[39] Reynolds P, Mohanty P, Pavic A. Use of operational modal analysis on empty and occupied stadia structures[C]//Proceedings of the 1st International Operational Modal Analysis Conference (IOMAC). 2005.

[40] Reynolds P, Pavic A. Impulse hammer versus shaker excitation for the modal testing of building floors[J]. Experimental Techniques, 2000, 24(3): 39-44.

[41] Sadhu A, Dunphy K, Zerpa C, et al. Investigation of vibration data-based human load monitoring system[J]. Structural Health Monitoring-an International Journal, 2019.

[42] Saudi G, Reynolds P, Zaki M, et al. Finite-element model tuning of global modes of a grandstand structure using ambient vibration testing[J]. Journal of Performance of Constructed Facilities, 2009, 23(6): 467-479.

[43] Severn R T. Structural Response Prediction Using Experimental Data: The sixth MalletMilne lecture[M]. CRC Press, 1997.

[44] Shahabpoor E, Pavic A, Racic V, et al. Structural Vibration Serviceability: New Design Framework Featuring Human-structure Interaction[J]. Engineering Structures, 2017: 295311.

[45] Shi W X, Wei D, Han R L. Field Tests and Research on the Dynamic Characteristics of Steel Structure Buildings Under Ambient Vibration [J]. Journal of Earthquake Engineering and Engineering Vibration, 2012,1(32):114-120. (In Chinese) 
[46] Smith A L, Hicks S J, Devine P J. Design of floors for vibration: A new approach[M]. Ascot, Berkshire, UK: Steel Construction Institute, 2007.

[47] Stoessel J C, Ibanez P, Keowen R S, et al. Design and Planned Use of a 2-Kiloton Eccentric Mass Vibrator[C]//Recent Advances in Structural Dynamics. ASCE, 1986: 34-39.

[48] Van Nimmen K, Lombaert G, De Roeck G, et al. The impact of vertical human-structure interaction on the response of footbridges to pedestrian excitation[J]. Journal of Sound and Vibration, 2017: 104-121.

[49] Ventura C E, Lord J F, Simpson R D. Effective use of ambient vibration measurements for modal updating of a 48 storey building in Vancouver, Canada[C]//International Conference on "Structural Dynamics Modeling-Test, Analysis, Correlation and Validation. 2002.

[50] Vukobratović V, Fajfar P. A method for the direct determination of approximate floor response spectra for SDOF inelastic structures[J]. Bulletin of Earthquake Engineering, 2015, 13(5): 1405-1424.

[51] Wang H, Nagayama T, Zhao B, et al. Identification of moving vehicle parameters using bridge responses and estimated bridge pavement roughness[J]. Engineering Structures, 2017, 153: 57-70.

[52] Willford M R, Young P, CEng M. A design guide for footfall induced vibration of structures[M]. Concrete Society for The Concrete Centre, 2006.

[53] Ying H Q, Liu J M, Shen S. Half-Power Bandwidth Method and INV Damping Ratio Solver Study[J]. Noise and Vibration Control, 2006(2). (In Chinese)

[54] Zhang M S, Chen J, Peng Y X, et al. Reproduction and simulation of walking induced load via motion capture technique and inverse dynamics of rigid body models[J]. Journal of Basic Science and Engineering, 2013, 21(5): 961-972. (In Chinese)

[55] Zhang M, Georgakis C T, Chen J, et al. Biomechanically Excited SMD Model of a Walking Pedestrian[J]. Journal of Bridge Engineering, 2016, 21(8).

[56] Zhou X H, Gao T T, Shi Y. Experimental Study on Static Deflection and Vibration Behaviour of Cold-Formed Steel OSB Composite Floor. Engineering Mechanics, 2014(05):211-217. (In Chinese) 